\title{
Anisotropic Electroplastic Effects on the Mechanical Properties of a Nano-Lamellar Austenitic Stainless Steel
}

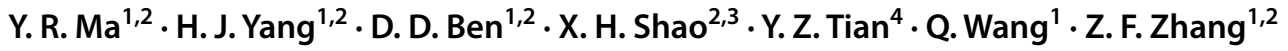

Received: 30 April 2020 / Revised: 14 June 2020 / Accepted: 24 June 2020 / Published online: 4 September 2020

(c) The Chinese Society for Metals (CSM) and Springer-Verlag GmbH Germany, part of Springer Nature 2020

\begin{abstract}
Effects of electropulsing treatment (EPT) on the microstructural evolution and mechanical properties of a cold-rolled 316L austenitic stainless steel with nano-lamellar structure were investigated. The EPT experiments were carried out with the electric current direction along the rolling direction (RD) and the transverse direction (TD) of the samples, respectively. Significant anisotropic electroplastic effects for the RD and TD specimens, i.e., reduced hardness/strength and enhanced ductility, were obtained owing to the different recrystallization behaviors of the RD and TD specimens during EPT. The RD specimens after EPT with larger recrystallized grain size and higher volume fraction of recrystallization show lower strength and higher elongation than that of the TD specimens. The main reason might be attributed to the change of the current direction in the two kinds of samples, which results in the different sensitivity of the microstructures to thermal and athermal effects during EPT.
\end{abstract}

Keywords Stainless steel $\cdot$ Electroplastic effect $\cdot$ Electropulsing $\cdot$ Recrystallization $\cdot$ Anisotropy $\cdot$ Strength $\cdot$ Plasticity

\section{Introduction}

Electropulsing treatment (EPT), as an instantaneous highenergy input method, was first discovered to be able to affect the performance of alloys in the 1960s [1,2]. EPT has been documented to induce the microstructural evolution and result in the corresponding outstanding performances

Available online at http://link.springer.com/journal/40195.

H. J. Yang

hjyang@imr.ac.cn

$\bowtie$ Z.F. Zhang

zhfzhang@imr.ac.cn

1 Shi-changxu Innovation Center for Advanced Materials, Institute of Metal Research, Chinese Academy of Sciences, Shenyang 110016, China

2 School of Materials Science and Engineering, University of Sciences and Technology of China, Hefei 230026, China

3 Shenyang National Laboratory of Materials Science, Institute of Metal Research, Chinese Academy of Sciences, Shenyang 110016, China

4 Key Laboratory for Anisotropy and Texture of Materials (Ministry of Education), School of Materials Science and Engineering, Northeastern University, Shenyang 110016, China of various metallic materials, such as recrystallization $[3$, 4], grain refining [5-7], hardening and strengthening [8, 9], enhancing fatigue performance $[10,11]$, corrosion resistance [12], crack healing [13-15] and so on. Meanwhile, EPT has been widely applied to materials processing, such as cold drawing, environmental protection, as well as in the medicine domain [16-18], which features a very short treatment time $[19,20]$. As a novel developing process, high-density pulse current in EPT has been proved to be an effective way to tune the microstructure and improve the mechanical properties of metallic materials.

The electroplastic (EP) effect is a phenomenon in which the deformation resistance of a material decreases sharply and the plasticity increases significantly when electric current passes through the material [21]. It was firstly reported by Troitskii in the study of zinc single crystal by uniaxial tensile in the 1960s [1]. From then on, many studies about electroplasticity were conducted to reveal the corresponding mechanisms [2, 22-24], and the related explanations primarily focused on the balance of two aspects, i.e., Joule heat of thermal effect and electron wind of athermal effect. However, the EP effects of EPT on the anisotropic microstructure of the materials are rarely reported and related information is also limited. 
In the present work, we chose $316 \mathrm{~L}$ austenitic stainless steel (316L SS) to unravel the mechanisms for the EPTinduced electroplasticity. 316L SS is one of the most versatile and corrosion-resistant alloys [25], and its tensile strength can reach up to $1.5 \mathrm{GPa}$ after $90 \%$ cold-rolling deformation. It is well known that cold-rolling deformation usually induces anisotropy of the specimens [26]. Namely, the microstructure, as well as the respective mechanical properties, along rolling direction and transverse direction is largely different from each other [27, 28]. Therefore, we aim to explore the microstructural evolution and mechanical properties in relation to the anisotropy of cold-rolled $316 \mathrm{~L}$ SS after EPT with different discharge voltages. And the possible mechanisms of the EPT-induced thermal and athermal effects on the recrystallization behaviors of the material are also discussed.

\section{Experimental}

A commercial 316L austenite stainless steel was used in this work. The as-received material was cold rolled (CR) at room temperature by multiple passed rolling from 40 to $4 \mathrm{~mm}$ with the total reduction of thickness $~ 90 \%$. As illustrated in Fig. 1, the EPT specimens having a gauge length of $40 \mathrm{~mm}$ and cross-sectional areas of $4 \times 6 \mathrm{~mm}$ were cut from the cold-rolled sheet along two directions to study the anisotropic EP effects: parallel to the rolling direction (RD) and perpendicular to the rolling direction (TD) by electrical discharge machining method. The TD and RD specimens with EPT are denoted as TD-EPT and RD-EPT hereafter, respectively. These CR-316L SS specimens were treated by EPT with various discharge voltages. EPT experiments were carried out on a self-built instrument based on an impulse capacitor with a single pulse in $400 \mathrm{~ns}$ through the sample. The surfaces of specimens were polished and cleaned before EPT experiments. Based on our previous study [8], the EPT discharge voltages were chosen as $12 \mathrm{kV}$ and $13 \mathrm{kV}$ in this work to magnify the anisotropy electroplasticity, which were referred to as EPT-12 and EPT-13, respectively.

Tensile tests were performed at room temperature using INSTRON 5982 testing machine operating at a strain rate of $10^{-3} \mathrm{~s}^{-1}$ for the as-received and EPT-processed specimens. The dog-bone-shaped tensile specimens were cut from the as-received and EPT-processed bars with a gauge section of $1 \times 3 \times 15 \mathrm{~mm}$ as indicated in Fig. 1b. The Vickers microhardness was measured by AMH43 automatic microindentation hardness testing system. To make an exact measurement, each specimen was measured 10 times with a load of $500 \mathrm{~g}$ for $13 \mathrm{~s}$ and the average value was defined as the measured hardness.

Transmission electron microscopy (TEM) was carried out on FEI Tecnai F20 operating at $200 \mathrm{kV}$. Thin foils for TEM observation were punched into disks of $3 \mathrm{~mm}$ in diameter,

(a)

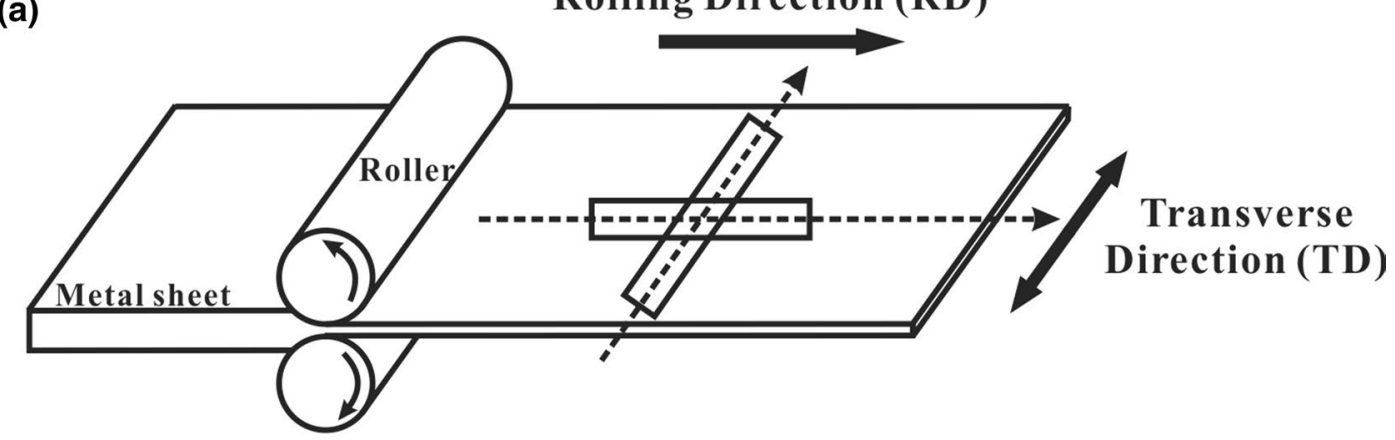

(b)

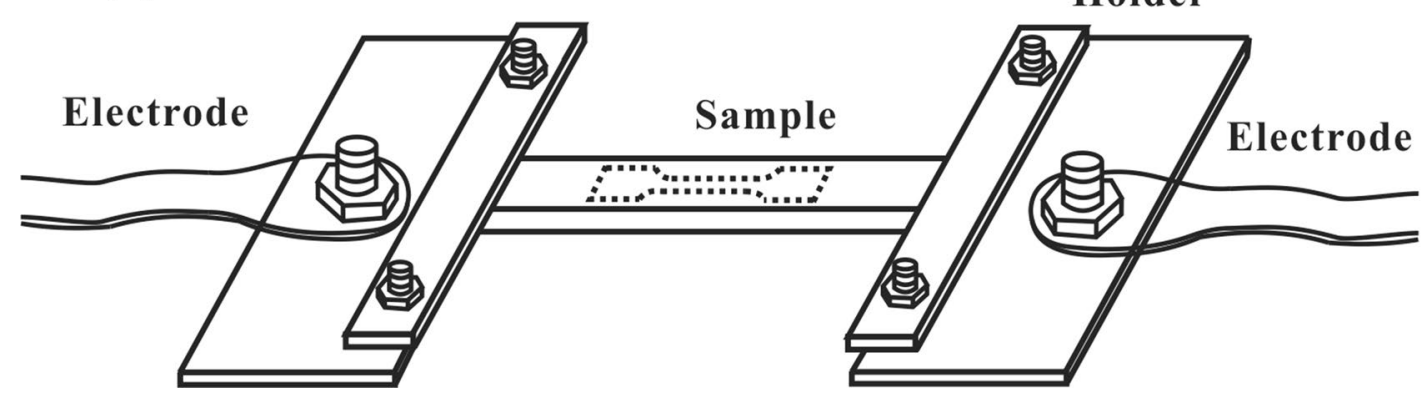

Fig. 1 Schematic diagram of the orientation of the CR-316L SS specimens a samples processed by EPT $\mathbf{b}$ 
mechanically polished to $\sim 50 \mu \mathrm{m}$, and then electrochemically polished in an electrolyte of $90 \mathrm{ml}$ ethanol, $10 \mathrm{ml}$ perchloric acid using a twin-jet electropolishing equipment at $-15{ }^{\circ} \mathrm{C}$ and an applied voltage of $28 \mathrm{~V}$. The grain size was calculated and estimated by a combination of the linear intercept method and TEM observations.

\section{Results}

\subsection{Microstructural Evolution of CR 316L SS Samples}

Figure 2 presents the typical microstructure of RD samples in CR-316L SS before and after EPT. The CR-316L SS features nanometer lamellar structure with $35 \mathrm{~nm}$ thickness before EPT, as shown in Fig. 2a. Also, dark contrast at the lamellar interface indicates profuse dislocations tangled $[8,29]$, as denoted by the arrows. Figure $2 b$ shows that the recrystallization nucleated at one end of lamellar grains in the RD-EPT-12 specimens, where the relatively higher energy may store. Figure $2 \mathrm{c}$ displays the partial recrystallization microstructure of the RD-EPT-13 specimens, i.e., one part keeps the original nanometer lamellar structure and the other part changes to the recrystallized grains with size from 100 to $800 \mathrm{~nm}$, and the average size is $\sim 500 \mathrm{~nm}$. That is, the recrystallized grain apparently grows larger with the increase in discharge voltage, and the volume fraction of recrystallized area is up to approximately $13 \%$.

It seems that the microstructural evolution of TD samples is similar to that of the RD samples. Figure $2 d$ shows that TD sample features nanometer lamellar structure and dense dislocations accumulated at the lamellar boundaries and tangled in the interior of the grains. Figure 2e presents that the nucleation of recrystallization formed at one end of the lamellar grains and also remained within the same lamellar grain in TD-EPT-12 sample. Whereas, the diameter of recrystallization grains in TD-EPT-12 sample is approximately $200 \mathrm{~nm}$, which is smaller than that of RDEPT-12 sample. As the pulse intensity increases to $13 \mathrm{kV}$ (Fig. 2f), a partial recrystallization structure was produced in TD-EPT-13 specimens, which is similar to that shown in Fig. 2c. The typical size of the recrystallized grains is around
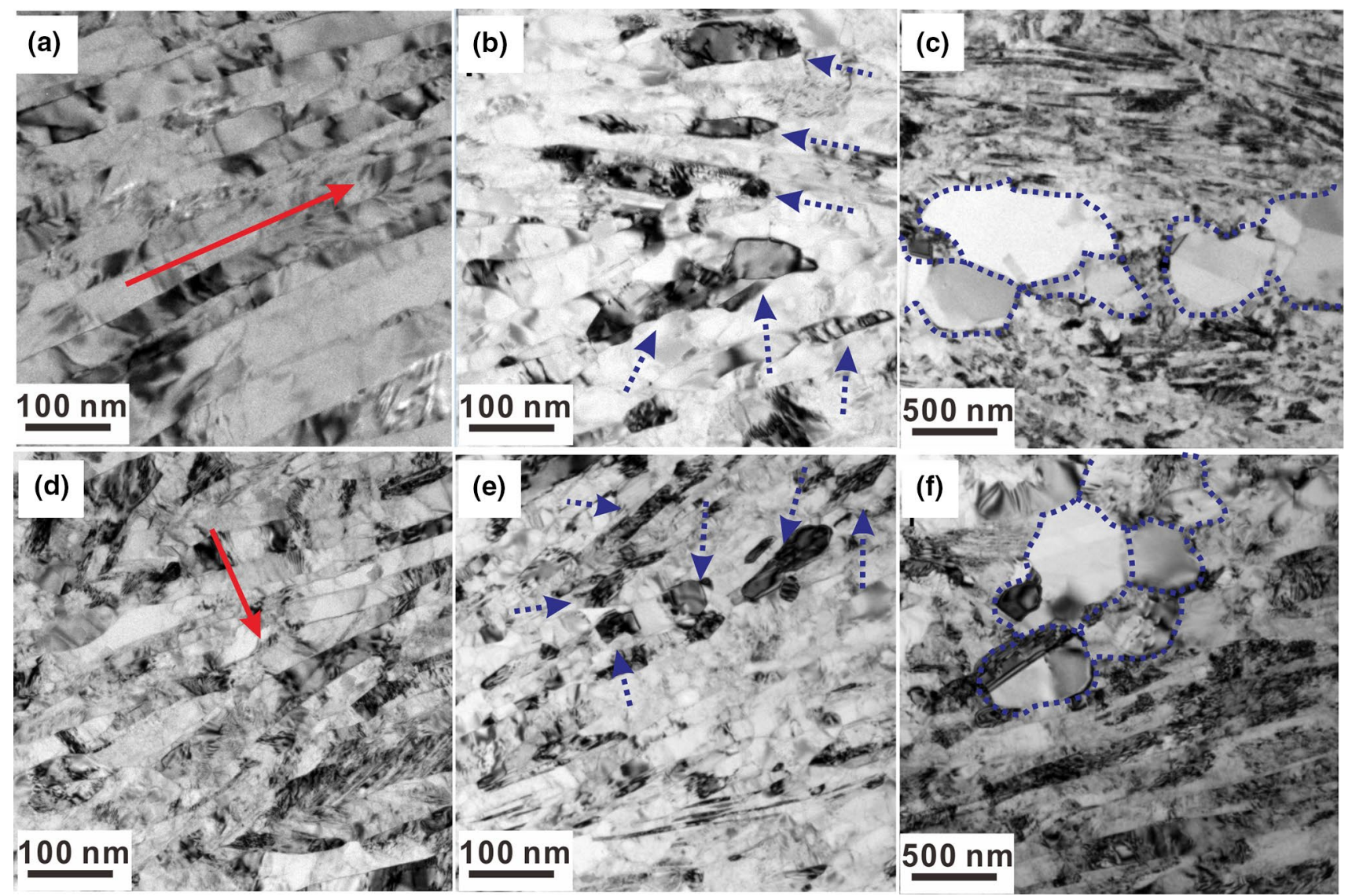

Fig. 2 Typical bright-field TEM images of the CR-316L SS samples after EPT with different discharge voltages: a RD, b RD-EPT12 kV, $\mathbf{c}$ RDEPT13 kV, d TD, e TD-EPT12 kV ,f TD-EPT13 kV 
$60-500 \mathrm{~nm}$ and the average size is $\sim 200 \mathrm{~nm}$, which is 2.5 times smaller than that in the RD-EPT-13 sample. Meanwhile, the recrystallization proportion in TD-EPT-13 specimens is about 7\%, which is half lower than that in the RDEPT-13 specimens. Therefore, the higher discharge voltage of EPT, the more distinguished effects on the microstructural anisotropy of RD and TD specimens, as far as the grain size and proportion of recrystallized grains are concerned.

\subsection{Mechanical Properties}

The microhardness of RD and TD samples before and after EPT is shown in Fig. 3. The microhardness of RD and TD samples is $407.4 \mathrm{HV}$ and $405.4 \mathrm{HV}$, respectively. The microhardness of RD-EPT and TD-EPT samples decreases with the increase in electron current intensity. Specifically, the microhardness of RD-EPT-13 sample decreased to 359.2 $\mathrm{HV}$ from that of RD sample of $407.4 \mathrm{HV}$ by $11.8 \%$, while the hardness of TD-EPT-13 samples decreased to $376.5 \mathrm{HV}$ from that of TD sample of $405.4 \mathrm{HV}$ by $7.1 \%$.

Figure 4 illustrates the tensile engineering stress-strain curves of the RD and TD specimens treated by EPT with different voltages. Obviously, one can find that the strength of the RD and TD samples decreases and the elongation increases with the increase in current intensity. Table 1 details the yield strength and elongation to failure of $316 \mathrm{~L}$ SS before and after EPT. As shown from the chart, after treated by $13 \mathrm{kV}$, the strength of the RD samples decreases from 1417 to $1046 \mathrm{MPa}$ and the elongation increases from 6.7 to $17.2 \%$. Similarly, the strength of TD-EPT-13 decreases from 1533 to $1292 \mathrm{MPa}$, and the elongation increases from 7.3 to $12.0 \%$. The EPT-induced softening of the specimens agrees well with the previous studies $[8,30]$, and it was rationalized by microstructure

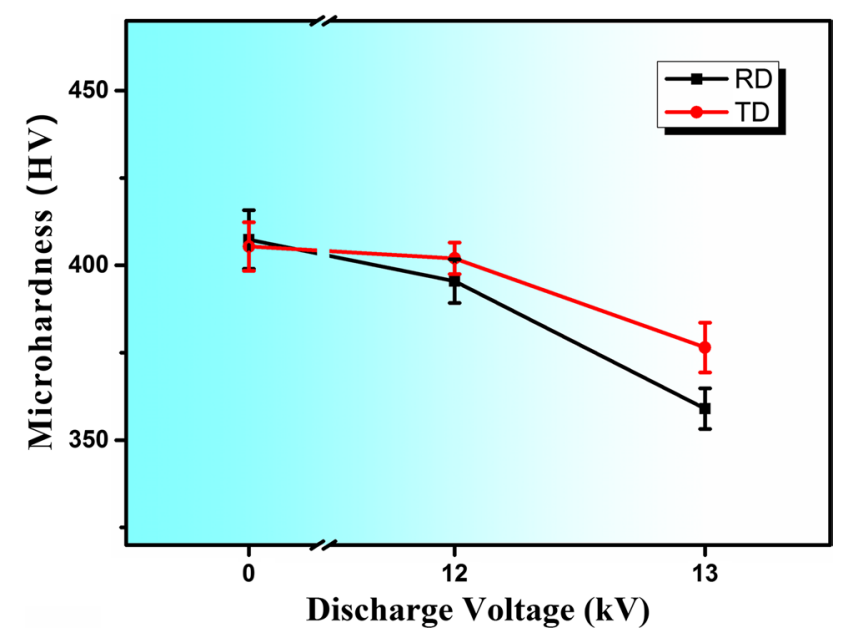

Fig. 3 Microhardness evolution of the CR-316L SS samples after EPT with different discharge voltages

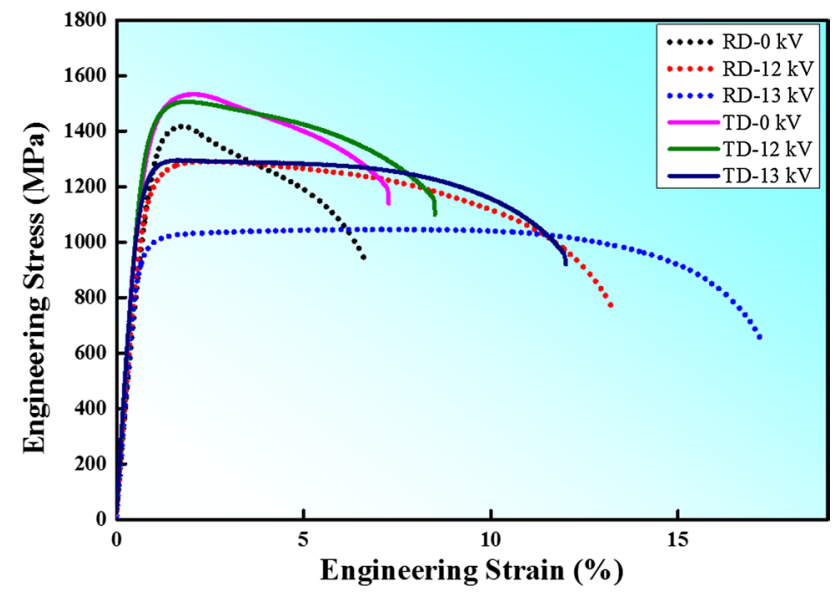

Fig. 4 Tensile engineering stress-strain curves of the CR-316L SS samples after EPT with different discharge voltages

recovery and recrystallization formed due to the high pulse intensities during EPT. Although the RD and TD samples showed the similar softening phenomenon, the obvious difference is that the electroplasticity of the RD samples is stronger under the same conditions. In other words, with the increase in pulse intensity, the strength of the RD sample decreases faster and the elongation increases more significantly.

To quantitatively describe the EP effect on the mechanical properties of CR-316L SS, we introduce the change ratio of strength and elongation of the RD and TD specimens under different EPT discharge voltages. The change ratio can be obtained according to the following equations [31]:

$\Delta \sigma=\frac{\sigma_{T}-\sigma_{0}}{\sigma_{T}} \times 100 \%$,

and

$\Delta \varepsilon=\frac{\varepsilon_{T}-\varepsilon_{0}}{\varepsilon_{T}} \times 100 \%$,

where $\Delta \sigma$ is the change ratio of strength, $\sigma_{\mathrm{T}}$ is the strength of the rolled samples with EPT, $\sigma_{0}$ is the strength of the rolled

Table 1 Strength and elongation of CR-316L SS before and after EPT

\begin{tabular}{lllll}
\hline Voltage $(\mathrm{kV})$ & $\begin{array}{l}\mathrm{RD} \\
\text { Yield } \\
\text { strength } \\
(\mathrm{MPa})\end{array}$ & $\begin{array}{l}\text { Elongation } \\
\text { to failure } \\
(\%)\end{array}$ & $\begin{array}{l}\text { TD } \\
\text { Yield } \\
\text { strength } \\
(\mathrm{MPa})\end{array}$ & $\begin{array}{l}\text { Elongation to } \\
\text { failure }(\%)\end{array}$ \\
\hline 0 & 1417 & 6.7 & 1533 & 7.3 \\
12 & 1291 & 13.2 & 1505 & 8.5 \\
13 & 1046 & 17.2 & 1292 & 12.0 \\
\hline
\end{tabular}


samples without EPT; $\Delta \varepsilon$ is the change ratio of elongation, $\varepsilon_{\mathrm{T}}$ is the elongation of the rolled samples with EPT, $\varepsilon_{0}$ is the elongation of the rolled samples without EPT.

Figure $5 \mathrm{a}, \mathrm{b}$ shows the percentage change ratio of strength and elongation of the CR-316L SS after EPT, respectively. The change ratio of strength of the RD specimen is about $-26.2 \%$ (1417 MPa of RD to $1046 \mathrm{MPa}$ of RD-EPT-13), while that of TD specimen is $-15.7 \%$ (1533 MPa of TD to $1292 \mathrm{MPa}$ of TD-EPT-13). That is, the EP effect on strength of the RD specimens is 1.67 times $(-26.2 /-15.7)$ higher than that of the TD specimens. On the contrary, the change ratio of elongation of the RD specimens is about $156.7 \%$ (6.7\% of RD to $17.2 \%$ of RD-EPT-13), while that of TD specimens is $64.4 \%$ (7.3\% of TD to $12 \%$ of TD-EPT-13). Namely, the EP effect on the elongation of the RD specimens is 2.43 times larger than that of the TD specimens. Thus, it is fair to say that the RD specimens experience stronger EP effects than the TD specimens on both the strength and plasticity. In addition, with the increase in EPT discharge voltage, the change ratio increases accordingly for both the $\mathrm{RD}$ and TD specimens, as indicated by the black arrows in Fig. 5.

The yield strength and elongation to failure for the EPTprocessed CR-316L SS samples are plotted in Fig. 6. It is found that the data present a traditional strength-ductility trade-off synergy. However, the EPT process was finished in microseconds which is much faster than that of the annealing process [32], indicating that EPT might have some advantages in optimizing the mechanical properties of severely deformed metals over the traditional heat treatment.

\section{Discussion}

The mechanical properties of the EPT-RD and EPT-TD specimens are different, as demonstrated in Figs. 3, 4, 5 and 6 , which are closely related to the characteristic microstructural evolutions. Specifically, EPT induced the anisotropic

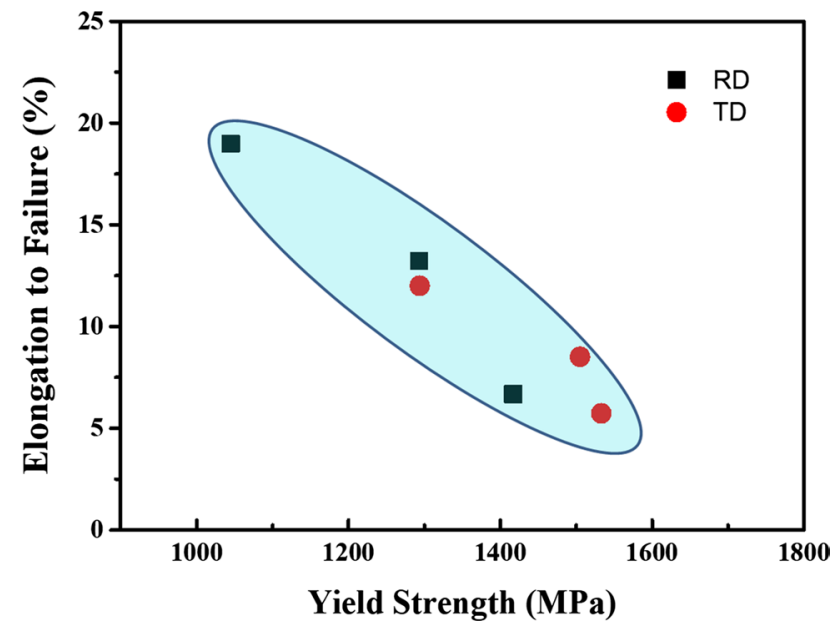

Fig. 6 Strength-ductility balance of the CR-316L SS after EPT

electroplasticity, i.e., the decrease in tensile strength and the increase in elongation, of the RD and TD samples with the increase in discharge voltage. The RD and TD samples distinguished from each other in the EPT-induced electron motion direction, as shown in Fig. 7. Figure 7a shows the three-dimensional sketch of the typical lamellar microstructure of the CR-316L SS. Figure 7b, c illustrates the EPT-induced electron motion in the RD and TD samples, respectively. In the following, we will rationalize the potential mechanisms based on the thermal and athermal effects of EPT on the RD and TD samples with different electron motion passes.

\subsection{Anisotropic Thermal and Athermal Effects on the RD and TD Specimens}

It is well established that a great number of high-rate electrons drift randomly during the passing of the electropulse through metallic material. Usually associated with the rambling motion of electrons, the collisions between
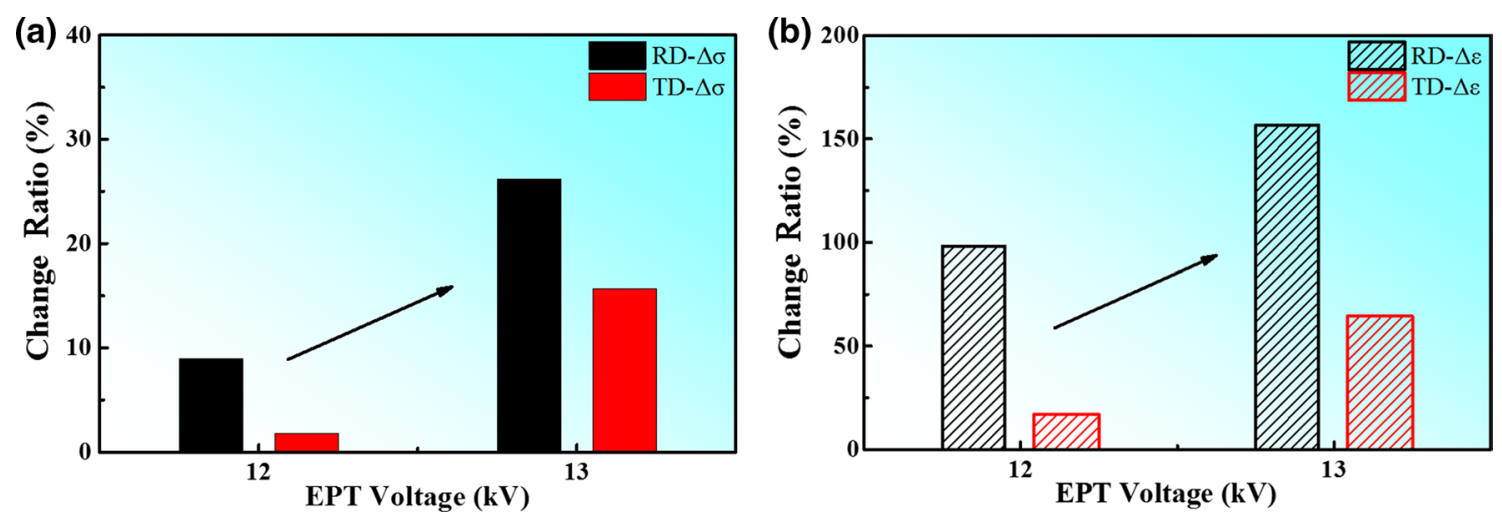

Fig. 5 Percentage change ratio of strength a and elongation $\mathbf{b}$ of CR-316L SS after EPT with different discharge voltages 


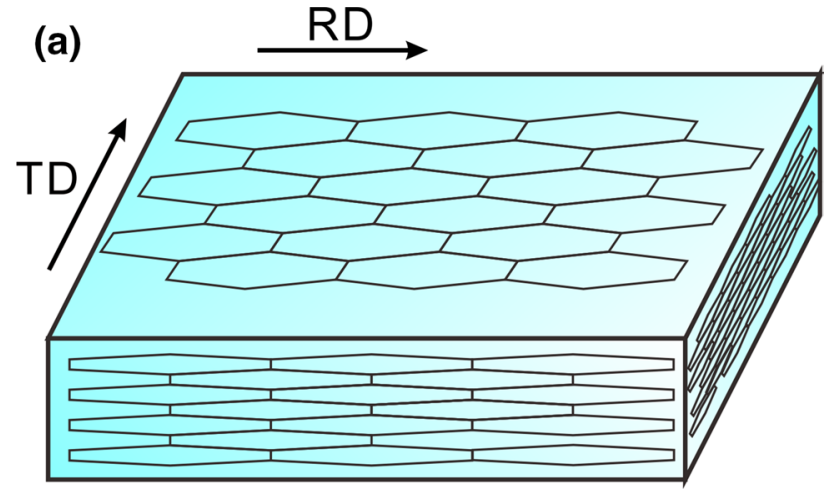

(b)

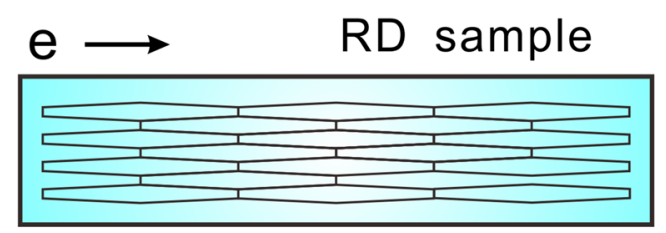

(c)

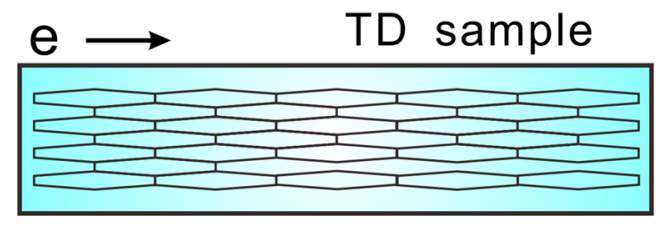

Fig. 7 Schematic diagram of typical lamellar microstructure of the CR-316L SS a, and EPT-induced electron motion in the RD $\mathbf{b}$ and TD samples $\mathbf{c}$

high-rate drift electrons and atomic nuclei will be converted into heat energy [33]. The heat energy, namely Joule heat, gives rise to the elevation of temperature of the material. The temperature increase induced by Joule heating can be calculated according to the following equation [34]:

$\Delta T=\frac{\int_{0}^{t} \rho_{e} I_{t}^{2} \mathrm{~d} t}{c \rho}=\frac{\int_{0}^{t} \rho_{e}\left(\frac{U_{t} S}{\rho_{e} l}\right)^{2} \mathrm{~d} t}{c \rho}=\frac{S^{2} \int_{0}^{t} U_{t}^{2} \mathrm{~d} t}{\rho_{e} c \rho l^{2}}$,

where $\Delta T$ is the increased temperature, $t$ is time, $\rho_{\mathrm{e}}$ is the electrical resistivity, $I_{\mathrm{t}}$ is instantaneous current value, $U_{\mathrm{t}}$ is instantaneous voltage value, $l$ is the length between the two electrodes, $S$ is the transverse area of sample, $c$ is specific heat capacity, $\rho$ is the density of material. For simplicity, we induced a parameter $A=\frac{S^{2} \int_{0}^{t} U_{t}^{2} \mathrm{~d} t}{c \rho l^{2}}$. In this way, only $\rho_{\mathrm{e}}$ is different for the EPT-12 and EPT-13 samples.

For the RD and TD samples, the current direction is basically parallel to the long axis of lamellar grain, while for the TD samples the long axis of the lamellar grain is obviously shorter than that of RD samples. It indicates that there are more grain boundaries in the TD samples per unit distance, which lead to a higher resistivity in the
TD samples. Namely, the electrical resistivity $\left(\rho_{\mathrm{e}}\right)$ of TD samples is larger than that of RD samples to some extent,

$\rho_{\mathrm{eRD}}<\rho_{\mathrm{eTD}}$.

Substituting Rel. 3 into Eq. (3), one can find that the temperature rise in the RD samples $\Delta T_{\mathrm{RD}}$ is higher than the temperature rise in the TD samples $\Delta T_{\mathrm{TD}}$, i.e.,

$\Delta T_{\mathrm{RD}}>\Delta T_{\mathrm{TD}}$.

In general, EPT-induced microstructural evolution of polycrystalline metallic materials is primarily due to the electron wind force generated by the electric current, which can scatter unevenly around dislocations and subsequently drives the dislocations to move within the lamellar grains and tangled at lamellar boundaries [35, 36], and which is also evident from the TEM observations in Fig. 8. As can be seen from Fig. 8a, the profuse dislocations uniformly distributed in the cold-rolled material. After EPT$12 \mathrm{kV}$, the dislocation density decreased significantly inside the lamellar grain, however, some dislocations were still observed at lamellar boundaries, as indicated by the red arrows in Fig. 8b. According to the resistance of dislocation per unit length, Troitskii et al. built a model for calculating the electronic wind force [37]:

$F_{\text {ew }}=\frac{\rho_{D}}{N_{D}} e n_{e} I_{t}=\frac{\rho_{D}}{N_{D}} e n_{e} \frac{U_{t}}{\rho_{e} l}$.

where $F_{\text {ew }}$ is the force applied to a unit dislocation length, $\rho_{\mathrm{D}} / N_{\mathrm{D}}$ is the unit dislocation resistance, $e$ is the electron charge, $n_{\mathrm{e}}$ is the electron density, $I_{\mathrm{t}}$ is the current density, $U_{\mathrm{t}}$ is the instantaneous voltage value, $l$ is the length between the two electrodes, $\rho_{\mathrm{e}}$ is the electrical resistivity.

As discussed above, the electrical resistivity $\left(\rho_{\mathrm{e}}\right)$ of the TD samples is higher than that of the RD samples because of the current direction differences.

Substituting Rel. 3 into Eq. (6), we obtain:

$F_{\text {ewRD }}>F_{\text {ewTD }}$.

Thus, the electronic wind force is higher in the EPT-RD samples than in the EPT-TD samples, which will trigger the dislocation motion faster. It has been reported that EPT can decrease the threshold for recrystallization nucleation, decrease the temperature of recrystallization, and accelerate the process of recrystallization nucleation [38]. In addition, EPT can tremendously accelerate the recrystallization process due to the substantial increase in the atomic flux resulted from the coupling of the thermal and athermal effects [39]. Therefore, because the thermal and athermal effects of RD samples are stronger, the recrystallization process of the EPT-RD samples should be faster, compared with the EPT-TD samples. 

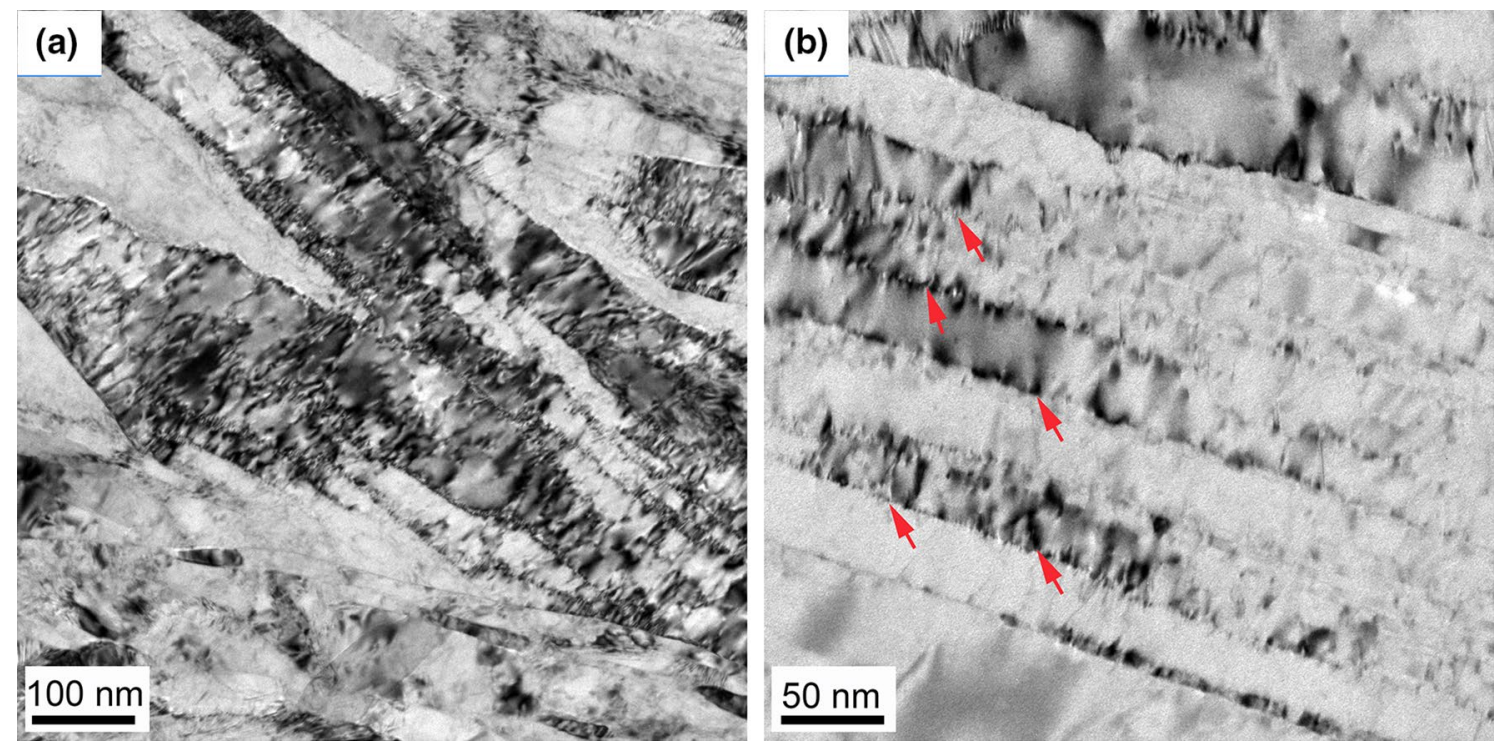

Fig. 8 Profuse dislocations distributed inside the lamellar grains and located at boundaries in the CR-316L SS sample a, educed dislocation density inside the lamellar grains while some dislocations tangled at lamellar boundaries as indicated by the red arrows in the RD-EPT12 kV sample b

It should be mentioned that there is a competition between the recrystallization nucleation and the new grain growth. It has been documented that the EPT process only takes several seconds for the cold-worked alloys to complete recrystallization, and the higher recrystallization rate was due to an athermal effect of EPT [40, 41]. When the current pulse intensity exceeds a certain level, the recrystallization process was dominated by thermal effect [42], which directly results in the coarsening of recrystallized grains. Taking the larger value of temperature increase and electron wind force, the recrystallization nucleation rate is fast, and the grains are larger in the EPT-RD samples.

\subsection{EPT induced the Decrease in Strength and Increase in Elongation}

After treated by EPT- $13 \mathrm{kV}$, because the temperature rise in the RD samples is higher than that of the TD samples as shown in Rel. (5), the recrystallized grains grow up and the average grain diameters are about $500 \mathrm{~nm}$ for the RD samples and $200 \mathrm{~nm}$ for the TD samples, respectively, which is approximately 10 times of the original lamella thickness $(\sim 35 \mathrm{~nm})$.

It is well known that the relationship between yield strength and grain size may be described mathematically by the Hall-Petch equation [43]:

$\sigma_{y}=\sigma_{0}+k_{y} / d^{-1 / 2}$,

where $\sigma_{\mathrm{y}}$ is yield strength, $\sigma_{0}$ is a material constant for the starting stress for dislocation movement (or the resistance of the lattice to dislocation motion), $k_{\mathrm{y}}$ is the strengthening coefficient and $d$ is the average grain diameter. According to the formula, the strength of metallic material is in the inverse proportional relationship to the square root of the average grain size. Then, the obvious growth of the recrystallized grains will significantly reduce the strength of the material and enhance the tensile plasticity. As a result, the strength and the elongation to failure exhibit $22.6 \%$ decrement and $156.7 \%$ increase for the EPT-RD-13 kV sample, respectively, while only $15.7 \%$ and $64.4 \%$ for the EPT-TD- $13 \mathrm{kV}$ sample, as compared with the CR-316L SS samples. And a similar tendency has been detected in the specimens treated by EPT at $12 \mathrm{kV}$.

In addition, austenite reversion after electropulsing treatment also played a role in electroplasticity. As shown in Fig. 9, a small amount of austenite was transformed into alpha'- martensite after severe plastic deformation. Since the RD and TD samples were taken from the same rolled plate, both contain the same amount of alpha'- martensite. With the increase in pulse intensity, the alpha'- martensite in both RD samples and TD samples transformed to austenite gradually, but the austenite reversion in RD samples was more obvious under the same parameters. As shown in the figure, the RD samples could hardly see the martensite peak after EPT- $13 \mathrm{kV}$, that is, the martensite was almost completely transformed into austenite. However, the TD samples could also see the obvious martensite peak after EPT- $13 \mathrm{kV}$, indicating that the austenite reversion was not completed. In a word, the different austenite reversion behaviors during EPT also suggest the stronger electroplasticity effect of the RD sample. 


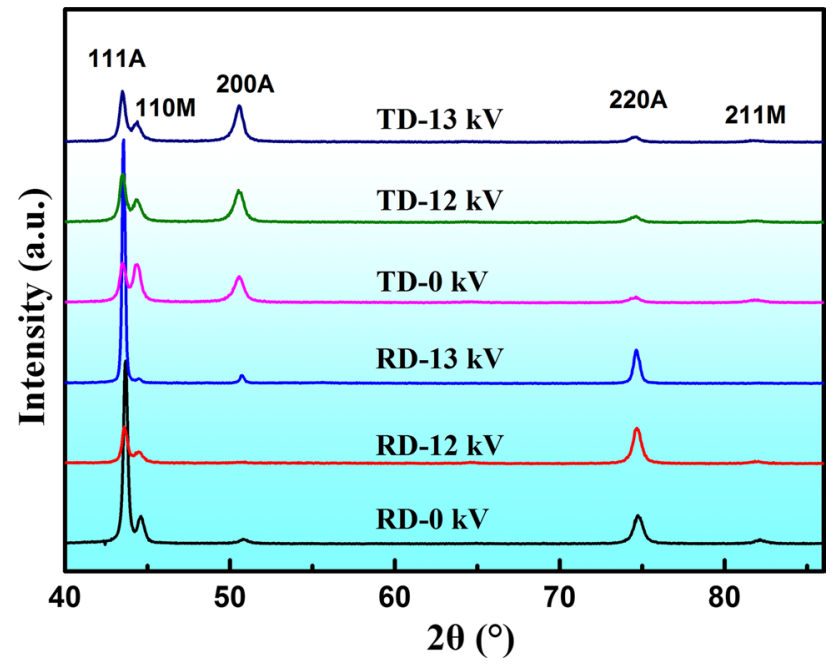

Fig. 9 XRD profiles of RD and TD samples treated by EPT with different discharge voltages

Combined with the microstructure and mechanical properties of the specimens before and after EPT, the EP effect of the RD samples caused by Joule heat and electron wind is more significant than that of TD samples. Moreover, Joule heat caused by thermal effect seems to surpass the electron wind force by athermal effect in the EPT-induced recrystallization process, i.e., the grain growth rate is faster than the nucleation rate. These new findings may be helpful for the optimization design of the microstructure and mechanical properties of the metallic materials after EPT and coldrolling treatments.

\section{Conclusions}

The microstructure and mechanical properties of a CR-316L SS after EPT along RD and TD were investigated. The following conclusions can be drawn:

1. Applying electropulsing treatment, the strength of the RD samples decreased faster than the TD samples, while the elongation increased faster, as the current intensity increased. The RD samples experienced stronger electroplasticity under instantaneous current.

2. The comprehensive action of thermal and athermal effect leads to the changes in microstructure and mechanical properties. The interaction between the electrons and dislocations of athermal effect decreases the threshold for recrystallization and accelerates the nucleate of recrystallization. What is more, Joule heat caused by thermal effect plays a major role in the recrystallized grain coarsening.
3. The different austenite reversion behaviors during EPT also suggest the stronger electroplasticity effect of the RD sample.

Acknowledgements This work was supported by the National Natural Science Foundation of China (Grants No. 51501196, 51975552 and 50711007), the IMR foundation for "Young merit scholars", the Youth Innovation Promotion Association CAS (No. 2017235) and the LiaoNing Revitalization Talents Program under Grant No. XLYC1808027.

\section{References}

[1] O.A. Troitskii, Sov. Phys. Dokl. 8, 277 (1963)

[2] O.A. Troitskii, A.G. Rozno, Fiz. Tverd. Tela 12, 203 (1969)

[3] Y.Z. Zhou, S.H. Xiao, J.D. Guo, Mater. Lett. 58, 1948 (2004)

[4] Y. Liu, J. Fan, H. Zhang, W. Jin, H. Dong, B. Xu, J. Alloys Compd. 622, 229 (2015)

[5] H. Song, Z.J. Wang, Trans. Nonferr. Metal. Soc. 21, 353 (2011)

[6] Y.Z. Zhou, W. Zhang, M.L. Sui, D.X. Li, G.H. He, J.D. Guo, J. Mater. Res. 17, 921 (2011)

[7] C. Wu, Y. Zhao, X.F. Xu, P. Yin, X. Qiu, Scr. Mater. 165, 6 (2019)

[8] Y.R. Ma, H.J. Yang, Y.Z. Tian, J.C. Pang, Z.F. Zhang, Mater. Sci. Eng., A 713, 146 (2018)

[9] D.D. Ben, H.J. Yang, Y.R. Ma, X.H. Shao, J.C. Pang, Z.F. Zhang, Mater. Sci. Eng., A 725, 28 (2018)

[10] H. Conrad, J. White, Mater. Sci. Eng., A 145, 1 (1991)

[11] D.D. Ben, H.J. Yang, Y.R. Ma, Q. Wang, Y.Z. Tian, P. Zhang, Q.Q. Duan, Z.F. Zhang, Adv. Eng. Mater. 21, 8 (2019)

[12] J.Y. Gao, X.B. Liu, H.F. Zhou, X.F. Zhang, Acta Metall. Sin. (Engl. Lett.) 31, 1233 (2018)

[13] Y.Z. Zhou, J.D. Guo, M. Gao, G.H. He, Mater. Lett. 58, 1732 (2004)

[14] H. Song, Z.J. Wang, Mater. Sci. Eng., A 490, 1 (2008)

[15] C.L. Yang, H.J. Yang, Z.J. Zhang, Z.F. Zhang, Scr. Mater. 147, 88 (2018)

[16] G.Y. Tang, J. Zhang, Y.J. Yan, H.H. Zhou, W. Fang, J. Mater. Process. Technol. 137, 96 (2003)

[17] R.S. Qin, B.L. Zhou, Int. J. Non-Equilib. Process. 11, 77 (1998)

[18] I. Sersa, K. Beravs, Magn. Reson. Med. 37, 404 (1997)

[19] Z. Xu, G. Tang, F. Ding, S. Tian, H. Tian, Appl. Phys. A 88, 429 (2007)

[20] R.F. Zhu, J.N. Liu, G.Y. Tang, S.Q. Shi, M.W. Fu, J. Alloys Compd. 544, 203 (2012)

[21] P. Song, X. Li, W. Ding, J. Chen, Acta Metall. Sin. (Engl. Lett.) 27, 642 (2014)

[22] Y. Onodera, K.I. Hirano, J. Mater. Sci. 11, 809 (1976)

[23] H. Conrad, A.F. Sprecher, W.D. Cao, X.P. Lu, JOM 42, 28 (1990)

[24] H. Xu, X. Liu, D. Zhang, X. Zhang, J. Mater. Sci. Technol. 35, 1108 (2019)

[25] K.H. Lo, C.H. Shek, J.K.L. Lai, Mater. Sci. Eng. R 65, 39-104 (2009)

[26] Y. Ma, Z.X. Du, X.M. Cui, J. Cheng, G.L. Liu, T.H. Gong, H.M. Liu, X.P. Wang, Y.Y. Chen, Prog. Nat. Sci. 28, 711 (2018)

[27] Z.J. Li, G. Winther, N. Hansen, Acta Mater. 54, 401 (2006)

[28] Z. You, H. Fu, S. Qu, W. Bao, L. Lu, Nano Mater. Sci. 2, 72 (2020)

[29] H. Miura, M. Kobayashi, Y. Todaka, C. Watanabe, Y. Aoyagi, N. Sugiura, N. Yoshiaga, Scr. Mater. 133, 33 (2017)

[30] S.H. Xiao, J.D. Guo, Scr. Mater. 46, 1 (2002)

[31] X.R. Chu, S.X. Lin, Z.M. Yue, J. Gao, C.S. Zhang, Mater. Sci. Technol. 31, 1601 (2015)

[32] F.K. Yan, G.Z. Liu, N.R. Tao, K. Lu, Acta Mater. 60, 1059 (2012) 
[33] W. Zhang, M.L. Sui, Y.Z. Zhou, D.X. Li, Micron 34, 189 (2003)

[34] H. Conrad, A.F. Sprecher, Dislocations in solids, edited by F.R.N. Nabarro, Amsterdam, Elservier, Chapter 43, 497 (1989)

[35] W.J. Lu, X.F. Zhang, R.S. Qin, Mater. Sci. Technol. 31, 1530 (2015)

[36] R.S. Qin, A. Rahnama, W.J. Lu, X.F. Zhang, Mater. Sci. Technol. 30, 1040 (2014)

[37] M.M. Moiseenko, O.A. Troitskii, Russ. Metall. 25, 159 (1987)

[38] G.L. Hu, Y.H. Zhu, G.Y. Tang, C.H. Shek, J.N. Liu, J. Mater. Sci. Technol. 27, 1034 (2011)
[39] Y. Jiang, G. Tang, C. Shek, W. Liu, J. Alloys Compd. 509, 4308 (2011)

[40] R.F. Zhu, G.Y. Tang, S.Q. Shi, M.W. Fu, V.E. Gromov, Appl. Phys. A 111, 1195 (2012)

[41] Z. Shan, J. Bai, J. Fan, H. Wu, H. Zhang, Q. Zhang, Y. Wu, W. Li, H. Dong, B. Xu, J. Mater. Sci. Technol. 51, 111 (2020)

[42] E.O. Hall, Proc. Phys. Soc. 64, 747 (1951)

[43] N.J. Petch, J. Iron Steel Inst. 173, 25 (1953) 